\title{
Correction: Data Sharing Goals for Nonprofit Funders of Clinical Trials
}

Timothy Coetzee ${ }^{1}$, MSc, PhD; Mad Price Ball ${ }^{2}$, PhD; Marc Boutin ${ }^{3}$, JD; Abby Bronson ${ }^{4}$, MBA; David T Dexter ${ }^{5}$, $\mathrm{PhD}$; Rebecca A English ${ }^{6}, \mathrm{MPH}$; Patricia Furlong ${ }^{7}$, BSN; Andrew D Goodman ${ }^{8}, \mathrm{MD}$; Cynthia Grossman ${ }^{9}, \mathrm{PhD}$; Adrian F Hernandez ${ }^{10}$, MD, MHS; Jennifer E Hinners ${ }^{11}$, MPH, MD; Lynn Hudson ${ }^{12}$, PhD; Annie Kennedy ${ }^{7}$, BSc; Mary Jane Marchisotto ${ }^{13}$, MBA; Lynn Matrisian ${ }^{14}, \mathrm{PhD}$, MBA; Elizabeth Myers ${ }^{15}$, PhD; W Benjamin Nowell ${ }^{16}$, PhD; Brian A Nosek ${ }^{17}, \mathrm{PhD}$; Todd Sherer ${ }^{18}, \mathrm{PhD}$; Carolyn Shore ${ }^{6}, \mathrm{PhD}$; Ida Sim ${ }^{19}, \mathrm{PhD}, \mathrm{MD}$; Luba Smolensky ${ }^{18}, \mathrm{BA}$; Christopher Williams $^{20}$, MSc; Julie Wood ${ }^{21}$, BA; Sharon F Terry ${ }^{22}$, MA, LhD

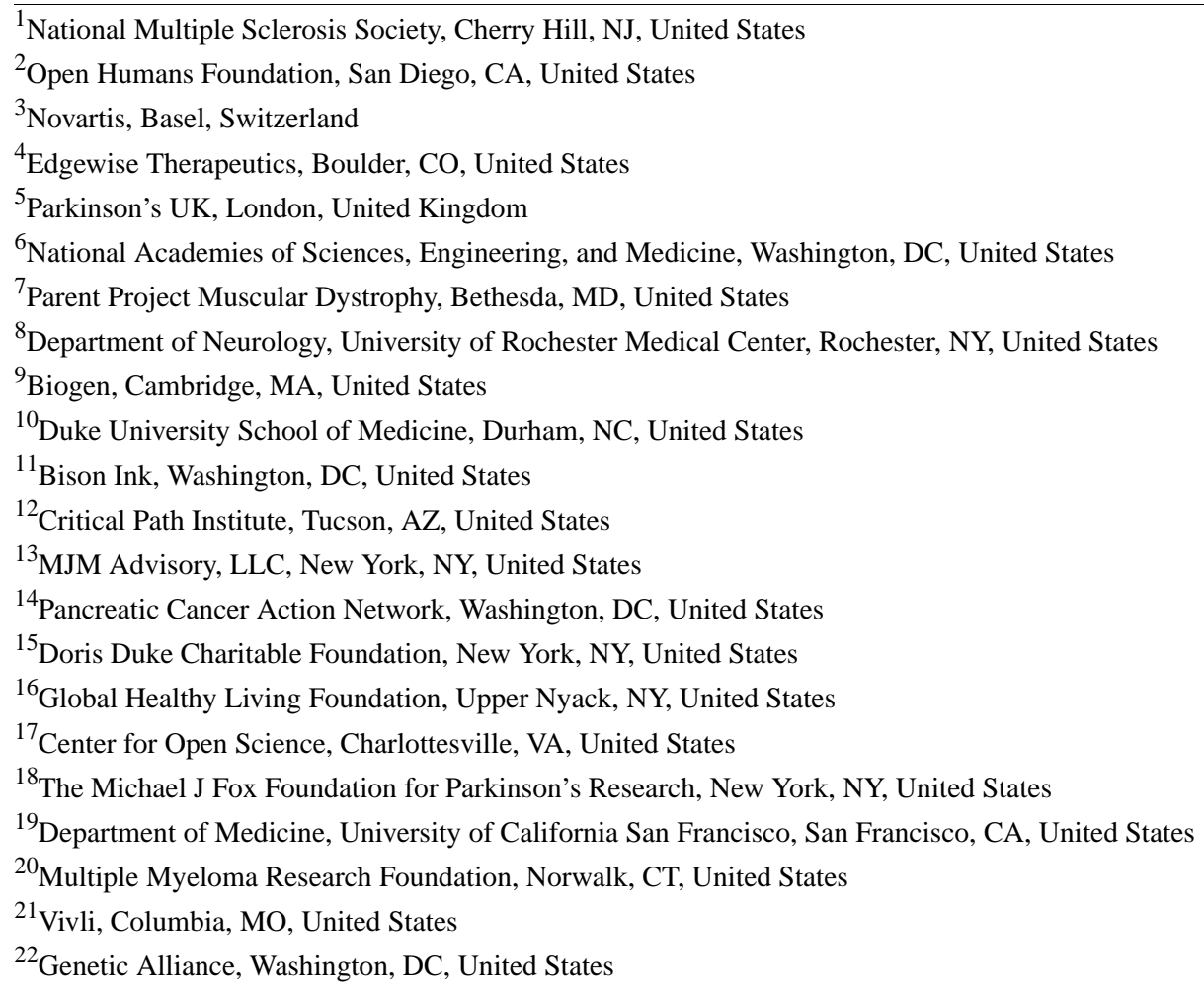

\section{Corresponding Author:}

Sharon F Terry, MA, LhD

Genetic Alliance

4301 Connecticut Ave NW \#404

Washington, DC

United States

Phone: 1 (202) 9665557

Email: sterry@geneticalliance.org

\section{Related Article:}

Correction of: https://jopm.jmir.org/2021/1/e23011

(J Particip Med 2021;13(2):e31371) doi: 10.2196/31371

In "Data Sharing Goals for Nonprofit Funders of Clinical Trials" (J Particip Med 2021;13(1):e23011) the authors noted two errors.

In the originally published manuscript, Lynn Matrisian was the last author listed in the order of authorship. This has been corrected to place Lynn Matrisian fifteenth in the order of authorship, after Mary Jane Marchisotto and before Elizabeth Myers. Author affiliations have been renumbered accordingly.

As well, the affiliation for Mary Jane Marchisotto has been corrected from:

MJM Advisory, LLC, McLean, VA, United States 
to:

MJM Advisory, LLC, New York, NY, United States

The complete list of authors and affiliations in the originally published paper was as follows:

Timothy Coetzee ${ }^{1}, \mathrm{MSc}$, PhD; Mad Price Ball ${ }^{2}, \mathrm{PhD}$; Marc Boutin ${ }^{3}$, JD; Abby Bronson ${ }^{4}$, MBA; David T Dexter ${ }^{5}$, PhD; Rebecca A English ${ }^{6}, M P H ;$ Patricia Furlong ${ }^{7}$, BSN; Andrew D Goodman ${ }^{8}, M D$; Cynthia Grossman $^{9}$, PhD; Adrian F Hernandez ${ }^{10}, M D, M H S$; Jennifer E Hinners ${ }^{11}$, MPH, MD; Lynn Hudson ${ }^{12}$, PhD; Annie Kennedy ${ }^{7}$, BSc; Mary Jane Marchisotto ${ }^{13}$, MBA; Elizabeth Myers ${ }^{14}$, PhD; W Benjamin Nowell ${ }^{15}$, PhD; Brian A Nosek ${ }^{16}$, PhD; Todd Sherer ${ }^{17}$, PhD; Carolyn Shore $^{6}$, PhD; Ida Sim ${ }^{18}$, PhD, MD; Luba Smolensky ${ }^{17}$, BA; Christopher Williams ${ }^{19}$, MSc; Julie Wood $^{20}$, BA; Sharon F Terry ${ }^{21}$, MA, LhD; Lynn Matrisian $^{22}, P h D, M B A$

${ }^{1}$ National Multiple Sclerosis Society, Cherry Hill, NJ, United States

${ }^{2}$ Open Humans Foundation, San Diego, CA, United States

${ }^{3}$ Novartis, Basel, Switzerland

${ }^{4}$ Edgewise Therapeutics, Boulder, CO, United States

${ }^{5}$ Parkinson's UK, London, United Kingdom

${ }^{6}$ National Academies of Sciences, Engineering, and Medicine, Washington, DC, United States

${ }^{7}$ Parent Project Muscular Dystrophy, Bethesda, MD, United States

${ }^{8}$ Department of Neurology, University of Rochester Medical Center, Rochester, NY, United States

${ }^{9}$ Biogen, Cambridge, MA, United States

${ }^{10}$ Duke University School of Medicine, Durham, NC, United States

${ }^{11}$ Bison Ink, Washington, DC, United States

${ }^{12}$ Critical Path Institute, Tucson, AZ, United States

${ }^{13}$ MJM Advisory, LLC, McLean, VA, United States

${ }^{14}$ Doris Duke Charitable Foundation, New York, NY, United States

${ }^{15}$ Global Healthy Living Foundation, Upper Nyack, NY, United States

${ }^{16}$ Center for Open Science, Charlottesville, VA, United States

${ }^{17}$ The Michael J Fox Foundation for Parkinson's Research, New York, NY, United States

${ }^{18}$ Department of Medicine, University of California San Francisco, San Francisco, CA, United States
${ }^{19}$ Multiple Myeloma Research Foundation, Norwalk, CT, United States

${ }^{20}$ Vivli, Columbia, MO, United States

${ }^{21}$ Genetic Alliance, Washington, DC, United States

${ }^{22}$ Pancreatic Cancer Action Network, Washington, $D C$, United States

The complete list of authors and affiliations in the corrected paper is as follows:

Timothy Coetzee ${ }^{1}, M S c, P h D ;$ Mad Price Ball ${ }^{2}, P h D ;$ Marc Boutin ${ }^{3}$, JD; Abby Bronson ${ }^{4}$, MBA; David T Dexter $^{5}$, PhD; Rebecca A English ${ }^{6}$, MPH; Patricia Furlong ${ }^{7}$, BSN; Andrew D Goodman ${ }^{8}, M D$; Cynthia Grossman $^{9}$, PhD; Adrian F Hernandez ${ }^{10}, M D, M H S$; Jennifer E Hinners ${ }^{11}$, MPH, MD; Lynn Hudson ${ }^{12}$, PhD; Annie Kennedy ${ }^{7}$, BSc; Mary Jane Marchisotto ${ }^{13}$, MBA; Lynn Matrisian ${ }^{14}$, PhD, MBA; Elizabeth Myers $^{15}$, PhD; W Benjamin Nowell ${ }^{16}$, PhD; Brian A Nosek $^{17}$, PhD; Todd Sherer ${ }^{18}$, PhD; Carolyn Shore ${ }^{6}$, PhD; Ida Sim $^{19}$, PhD, MD; Luba Smolensky ${ }^{18}, B A$; Christopher Williams ${ }^{20}$, MSc; Julie Wood ${ }^{21}$, BA; Sharon F Terry ${ }^{22}$, MA, LhD

${ }^{I}$ National Multiple Sclerosis Society, Cherry Hill, NJ, United States

${ }^{2}$ Open Humans Foundation, San Diego, CA, United States

${ }^{3}$ Novartis, Basel, Switzerland

${ }^{4}$ Edgewise Therapeutics, Boulder, CO, United States

${ }^{5}$ Parkinson's UK, London, United Kingdom

${ }^{6}$ National Academies of Sciences, Engineering, and Medicine, Washington, DC, United States

${ }^{7}$ Parent Project Muscular Dystrophy, Bethesda, MD, United States

${ }^{8}$ Department of Neurology, University of Rochester Medical Center, Rochester, NY, United States

${ }^{9}$ Biogen, Cambridge, MA, United States

${ }^{10}$ Duke University School of Medicine, Durham, NC, United States

${ }^{11}$ Bison Ink, Washington, DC, United States

${ }^{12}$ Critical Path Institute, Tucson, AZ, United States

${ }^{13}$ MJM Advisory, LLC, New York, NY, United States

${ }^{14}$ Pancreatic Cancer Action Network, Washington, DC, United States

${ }^{15}$ Doris Duke Charitable Foundation, New York, NY, United States

${ }^{16}$ Global Healthy Living Foundation, Upper Nyack, NY, United States 
${ }^{17}$ Center for Open Science, Charlottesville, VA, United States

${ }^{18}$ The Michael J Fox Foundation for Parkinson's Research, New York, NY, United States

${ }^{19}$ Department of Medicine, University of California San Francisco, San Francisco, CA, United States

${ }^{20}$ Multiple Myeloma Research Foundation, Norwalk, CT, United States

\author{
${ }^{21}$ Vivli, Columbia, MO, United States \\ ${ }^{22}$ Genetic Alliance, Washington, DC, United States
}

The correction will appear in the online version of the paper on the JMIR Publications website on June 30, 2021, together with the publication of this correction notice. Because this was made after submission to PubMed, PubMed Central, and other full-text repositories, the corrected article has also been resubmitted to those repositories.

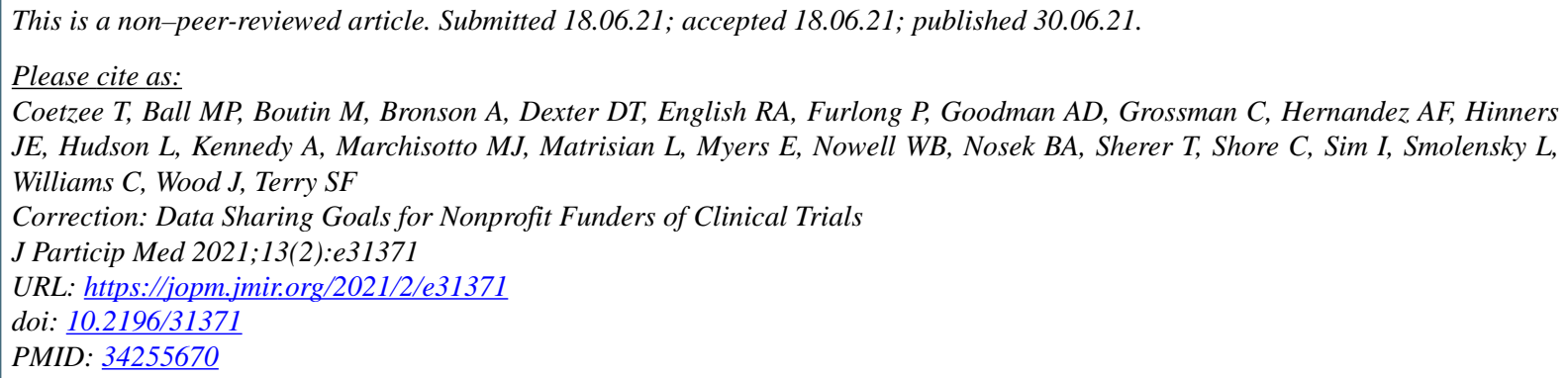

(C) Timothy Coetzee, Mad Price Ball, Marc Boutin, Abby Bronson, David T Dexter, Rebecca A English, Patricia Furlong, Andrew D Goodman, Cynthia Grossman, Adrian F Hernandez, Jennifer E Hinners, Lynn Hudson, Annie Kennedy, Mary Jane Marchisotto, Lynn Matrisian, Elizabeth Myers, W Benjamin Nowell, Brian A Nosek, Todd Sherer, Carolyn Shore, Ida Sim, Luba Smolensky, Christopher Williams, Julie Wood, Sharon F Terry. Originally published in Journal of Participatory Medicine (https://jopm.jmir.org), 30.06.2021. This is an open-access article distributed under the terms of the Creative Commons Attribution License (https://creativecommons.org/licenses/by/4.0/), which permits unrestricted use, distribution, and reproduction in any medium, provided the original work, first published in Journal of Participatory Medicine, is properly cited. The complete bibliographic information, a link to the original publication on https://jopm.jmir.org, as well as this copyright and license information must be included. 